\title{
A Series Solution for Three-Dimensional Navier-Stokes Equations of Flow near an Infinite Rotating Disk
}

\author{
Hamed Shahmohamadi*, Mahdi Mohammadpour \\ Wolfson School of Mechanical and Manufacturing Engineering, Loughborough University, Loughborough, UK \\ Email: ${ }^{*}$ h.shahmohamadi@lboro.ac.uk
}

Received 22 January 2014; revised 25 February 2014; accepted 21 March 2014

Copyright (C) 2014 by authors and Scientific Research Publishing Inc.

This work is licensed under the Creative Commons Attribution International License (CC BY). http://creativecommons.org/licenses/by/4.0/

c) (i) Open Access

\begin{abstract}
In this paper, homotopy analysis method (HAM) and Padé approximant will be considered for finding analytical solution of three-dimensional viscous flow near an infinite rotating disk which is a well-known classical problem in fluid mechanics. The solution is compared to the numerical (fourth-order Runge-Kutta) solution and the convergence of the obtained series solution is carefully analyzed. The results illustrate that HAM-Padé is an appropriate method in solving the systems of nonlinear equations.
\end{abstract}

\section{Keywords}

Homotopy Analysis Method, Padé Approximant, Navier-Stokes Equations, Rotating Disk

\section{Introduction}

Von Karman swirling viscous flow [1] is a well-known classical problem in fluid mechanics. The original problem raised by Von Karman is about the viscous flow induced by an infinite rotating disk where the fluid, far from the disk, is at rest. Then the problem is generalized in considering the case where the fluid itself is rotating as a solid body far from the disk with suction or injection at the disk surface [2]. This generates a parameter, i.e. the ratio of the angular velocity of the fluid at infinity to the angular velocity of the disk. Another generalization is to consider the viscous flow between two infinite coaxial rotating disks with suction or injection at both disks and this reveals another parameter, i.e. the Reynolds number determined by the distance of the two disks.

Nonlinear phenomena play a crucial role in applied mathematics and physics. It is known that most of engineering problems are nonlinear and solving them analytically is very difficult. Various powerful mathematical

\footnotetext{
${ }^{*}$ Corresponding author.
}

How to cite this paper: Shahmohamadi, H. and Mohammadpour, M. (2014) A Series Solution for Three-Dimensional Navier-Stokes Equations of Flow near an Infinite Rotating Disk. World Journal of Mechanics, 4, $117-127$. 
methods such as inverse scattering method [3], tanh method [4], sine-cosine method [5], homogeneous balance method [6], Exp-function method [7] and variational methods [8]-[12] have been proposed for obtaining exact and approximate analytic solutions. Recently, two analytical techniques have drawn specific attention, namely homotopy analysis method (HAM) and homotopy perturbation method (HPM). The essential idea in these methods is introducing a homotopy parameter, like $p$, which takes the value from 0 to 1 . For $p=0$, the system of equations takes a simplified form, which readily admits a particularly simple solution. When $p$ is gradually being increased to 1 , the system goes through a sequence of "deformations", the solution of each of which is "close" to that at the previous stage of "deformation". Eventually at $p=1$ the system takes the original form of equation and the final stage of "deformation" gives the desired solution. In 1992, Liao [13] [14] has been the leading exponent of homotopy analysis method (HAM). In HAM, Liao keeps sufficient room for experimenting the convergence of approximations by introducing auxiliary parameter and also, an auxiliary non-zero function. Another way of viewing homotopy approach is to think of $p$ as a perturbation parameter. In 1998, He [15] has transformed this idea into homotopy perturbation method (HPM). The approximate solution given by HAM is the same as the approximate solution given by HPM, when auxiliary parameter and auxiliary non-zero function are taken -1 and 1, respectively [16]-[19]. So, the approximate analytical solution obtained by HAM contains the solution obtained by HPM. However, the implementation of these methods shows that HAM is more effective than HPM. The convergence of HAM solution series depends on four factors, i.e. initial guess, auxiliary linear operator, auxiliary non-zero function and auxiliary parameter $\hbar$. However, as a special case of homotopy analysis method by having $\hbar=-1$, the convergence of HPM solution series only depends on two factors: the auxiliary linear operator and the initial guess. Therefore, having the initial guess and the auxiliary linear operator, HPM cannot provide other ways to ensure that the solution is convergent. Note that the HAM has already been applied to the analytical solution of several other problems [20]-[22].

Ismail and Rabboh [23] presented a restrictive Padé approximation for the generalized Fisher and Burger-Fisher equations. The Padé approximants that often show superior performance over series approximations provide a successful tool and promising scheme for identical applications.

The purpose of this paper is to extend homotopy analysis method and Padé approximant to solve three-dimensional Navier-Stokes equations for the viscous flow near an infinite rotating disk.

\section{Flow Analysis and Mathematical Formulation}

The Navier-Stokes equations for cylindrical coordinate are as follows:

$$
\begin{gathered}
\frac{1}{r} \frac{\partial}{\partial r}(r u)+\frac{1}{r} \frac{\partial}{\partial \theta}(v)+\frac{\partial w}{\partial z}=0 \\
\rho\left[\frac{\partial u}{\partial t}+u \frac{\partial u}{\partial r}+\frac{1}{r} v \frac{\partial u}{\partial \theta}+w \frac{\partial u}{\partial z}-\frac{v^{2}}{r}\right]+\frac{\partial p}{\partial r}=\mu\left[\frac{\partial^{2} u}{\partial r^{2}}+\frac{1}{r} \frac{\partial u}{\partial r}+\frac{1}{r^{2}} \frac{\partial^{2} u}{\partial \theta^{2}}+\frac{\partial^{2} u}{\partial z^{2}}-\frac{u}{r^{2}}-\frac{2}{r^{2}} \frac{\partial v}{\partial \theta}\right]+\rho g_{r} \\
\rho\left[\frac{\partial v}{\partial t}+u \frac{\partial v}{\partial r}+\frac{1}{r} v \frac{\partial v}{\partial \theta}+w \frac{\partial v}{\partial z}+\frac{u v}{r}\right]+\frac{1}{r} \frac{\partial p}{\partial \theta}=\mu\left[\frac{\partial^{2} v}{\partial r^{2}}+\frac{1}{r} \frac{\partial v}{\partial r}+\frac{1}{r^{2}} \frac{\partial^{2} v}{\partial \theta^{2}}+\frac{\partial^{2} v}{\partial z^{2}}-\frac{v}{r^{2}}+\frac{2}{r^{2}} \frac{\partial u}{\partial \theta}\right]+\rho g_{\theta} \\
\rho\left[\frac{\partial w}{\partial t}+u \frac{\partial w}{\partial r}+\frac{1}{r} v \frac{\partial w}{\partial \theta}+w \frac{\partial w}{\partial z}\right]+\frac{\partial p}{\partial z}=\mu\left[\frac{\partial^{2} w}{\partial r^{2}}+\frac{1}{r} \frac{\partial w}{\partial r}+\frac{1}{r^{2}} \frac{\partial^{2} w}{\partial \theta^{2}}+\frac{\partial^{2} w}{\partial z^{2}}\right]+\rho g_{z}
\end{gathered}
$$

where $u, v$ and $w$ are velocities in the $r, \theta$ and $z$ directions; respectively. $\rho, p$ and $\mu$ are density, pressure and viscosity. Consider the steady flow which results if the infinite plane $z=0$ rotates at constant angular velocity $\omega$ about the axis $r=0$ beneath a Newtonian viscous fluid which otherwise be at rest. The viscous drag of the rotating surface would set up a swirling flow toward the disk. All three velocity components $u, v$ and $w$ would be involved in a genuine three-dimensional motion, but because of radial symmetry they will be independent of $\theta$ as would the pressure $p$, so it is required to solve the equation for these four variables as functions of $r$ and $z$ from the continuity equation and the momentum equations in the $r, \theta$ and $z$ directions we obtain

$$
\frac{1}{r} \frac{\partial(r u)}{\partial r}+\frac{\partial w}{\partial z}=0
$$




$$
\begin{array}{r}
\rho\left[u \frac{\partial u}{\partial r}+w \frac{\partial u}{\partial z}-\frac{v^{2}}{r}\right]+\frac{\partial p}{\partial r}=\mu\left[\frac{\partial^{2} u}{\partial r^{2}}+\frac{1}{r} \frac{\partial u}{\partial r}+\frac{\partial^{2} u}{\partial z^{2}}-\frac{u}{r^{2}}\right] \\
\rho\left[u \frac{\partial v}{\partial r}+w \frac{\partial v}{\partial z}+\frac{u v}{r}\right]=\mu\left[\frac{\partial^{2} v}{\partial r^{2}}+\frac{1}{r} \frac{\partial v}{\partial r}+\frac{\partial^{2} v}{\partial z^{2}}-\frac{v}{r^{2}}\right] \\
\rho\left[u \frac{\partial w}{\partial r}+w \frac{\partial w}{\partial z}\right]+\frac{\partial p}{\partial z}=\mu\left[\frac{\partial^{2} w}{\partial r^{2}}+\frac{1}{r} \frac{\partial w}{\partial r}+\frac{\partial^{2} w}{\partial z^{2}}\right]
\end{array}
$$

The boundary conditions are no-slip at the disk wall and inviscid flow far from the disk (except an axial inflow):

$$
\begin{gathered}
u=w=p=0, v=r \omega \text { at } z=0 \\
u=v=0 \text { at } z \rightarrow \infty
\end{gathered}
$$

Note that we refrain from imposing any conditions on $w$ as $z \rightarrow \infty$, because we expect (by hindsight, to be sure) that the disk will have a centrifugal pumping action which must be balanced by a uniform axial inflow $w<0$ far from the disk. Also the condition $p=0$ (a convenient constant) at the wall is by hindsight. It has been deduced that $u / r, v / r, w$ and $p$ are all functions of $z$. Since the only two parameters in the problem are $v$ and $\omega$, it is easy to see that the proper dimensionless variable must be $t=z \sqrt{\omega / v}, v=\mu / \rho$. Regarding Karman (1921), we propose the new dimensionless variables $f, g, h$ and $k$ as

$$
u=r \omega f(t), v=r \omega g(t), w=\sqrt{v \omega} h(t), p=\rho v \omega k(t)
$$

These variables are substituted into Equations (5)-(8) and the following set of nonlinear ordinary coupled differential equations is obtained:

$$
\begin{gathered}
f^{\prime \prime}(t)-f^{2}(t)+g^{2}(t)-f^{\prime}(t) h(t)=0, \quad f(0)=f(\infty)=0 \\
g^{\prime \prime}(t)-2 f(t) g(t)-g^{\prime}(t) h(t)=0, \quad g(0)=1, \quad g(\infty)=0 \\
h^{\prime}(t)+2 f(t)=0, \quad h(0)=0 \\
k^{\prime}(t)-2 f(t) h(t)+2 f^{\prime}(t)=0, \quad k(0)=0
\end{gathered}
$$

\section{Basic Concepts of HAM}

Consider the following differential equation

$$
N[u(\tau)=0],
$$

where $N$ is a nonlinear operator, $\tau$ denotes an independent variable, $u(\tau)$ is an unknown function which is the solution for the equation. The function is defined as

$$
\underset{p \rightarrow 0}{\varphi(\tau ; p)}=u_{0}(\tau),
$$

and

$$
\operatorname{Lim}_{p \rightarrow 1} \varphi(\tau ; p)=u(\tau) .
$$

where, $p \in[0,1]$ and $u_{0}(\tau)$ are the initial guesses which satisfy the initial or boundary condition.

By means of generalizing the traditional homotopy method, Liao [13] forms the so-called zero-order deformation equation as follows

$$
(1-p) L\left[\varphi(\tau ; p)-u_{0}(\tau)\right]=p \hbar H(\tau) N[\varphi(\tau ; p)],
$$

where $\hbar$ is the auxiliary parameter which increases the convergence of result, $H(\tau) \neq 0$ is an auxiliary function and $L$ is an auxiliary linear operator, $p$ increases from 0 to 1 , the solution $\phi(\tau ; p)$ changes between the initial guess $u_{0}(\tau ; p)$ and solution $u(\tau)$. Expanding $\phi(\tau ; p)$ in Taylor series with respect to $p$, we ob- 
tain

$$
\varphi(\tau ; p)=u_{0}(\tau)+\sum_{m=1}^{\infty} u_{m}(\tau) p^{m}
$$

where

$$
u_{m}(\tau)=\left.\frac{1}{m !} \frac{\partial^{m} \phi(\tau ; p)}{\partial p^{m}}\right|_{p=o}
$$

if the auxiliary linear operator, the initial guess, the auxiliary parameter $\hbar$ and the auxiliary function are so properly chosen, the series (20) converges at $p=1$, so we have

$$
u(\tau)=u_{0}(\tau)+\sum_{m=1}^{\infty} u_{m}(\tau)
$$

which must be one of the solutions of the original nonlinear equation, as proved by Liao [13]. It is clear that if the auxiliary parameter is $\hbar=-1$, and auxiliary function is determined to be $H(\tau)=1$, Equation (19) would be

$$
(1-p) L\left[\varphi(\tau ; p)-u_{0}(\tau)\right]+p N[\varphi(\tau ; p)]=0
$$

This statement is commonly used in HPM procedure. Indeed, in HPM, the nonlinear differential equation is solved by separating every Taylor expansion term. Now, the vector of $\boldsymbol{u}_{m}$ is defined as

$$
\boldsymbol{u}_{m}=\left\{\boldsymbol{u}_{1}, \boldsymbol{u}_{2}, \boldsymbol{u}_{3}, \cdots, \boldsymbol{u}_{n}\right\}
$$

according to the definition Equation (21), the governing equation and the corresponding initial condition of $u_{m}(\tau)$ can be deduced from zero-order deformation Equation (19). Differentiating Equation (19), $m$ times with respect to the embedding parameter $p$ and setting $p=0$ and finally dividing by $m$ !, we will have the socalled $m$ th order deformation equation in the following from

$$
L\left[u_{m}(\tau)-x_{m} u_{m-1}\right]=\hbar H(\tau) R_{m}\left(\boldsymbol{u}_{m-1}\right),
$$

where

$$
R_{m}\left(\boldsymbol{u}_{m-1}\right)=\left.\frac{1}{(m-1) !} \frac{\partial^{m-1} N[\varphi(\tau ; p)]}{\partial p^{m-1}}\right|_{p=0}
$$

and

$$
x_{m}= \begin{cases}0 & m \leq 1, \\ 1 & m>1 .\end{cases}
$$

Therefore by applying inverse linear operator to both sides of the linear equation, Equation (25), we can easily solve the equation and compute the generation constant by applying the initial or boundary conditions.

\section{HAM-Padé Solution}

In this section the HAM is used to find approximate solutions of the Equations (12)-(15). We choose the initial approximation as

$$
f_{o}(t)=0, g_{o}(t)=0, h_{o}(t)=0, k_{o}(t)=0,
$$

and the linear operator for Equations (12) to (15)

$$
\begin{aligned}
& L\left[\phi_{1}(t ; p)\right]=\frac{\partial^{2} \phi_{1}(t ; p)}{\partial t^{2}}, \\
& L\left[\phi_{2}(t ; p)\right]=\frac{\partial^{2} \phi_{2}(t ; p)}{\partial t^{2}},
\end{aligned}
$$




$$
\begin{aligned}
& L\left[\phi_{3}(t ; p)\right]=\frac{\partial \phi_{3}(t ; p)}{\partial t}, \\
& L\left[\phi_{4}(t ; p)\right]=\frac{\partial \phi_{4}(t ; p)}{\partial t} .
\end{aligned}
$$

We convert Equations (12) through (15) to nonlinear form:

$$
\begin{gathered}
N_{1}\left[\phi_{1}(t ; p), \phi_{2}(t ; p), \phi_{3}(t ; p)\right]=\frac{\partial^{2} \phi_{1}(t ; p)}{\partial t^{2}}-\phi_{1}^{2}(t ; p)+\phi_{2}^{2}(t ; p)-\frac{\partial \phi_{1}(t ; p)}{\partial t} \phi_{3}(t ; p) \\
N_{2}\left[\phi_{1}(t ; p), \phi_{2}(t ; p), \phi_{3}(t ; p)\right]=\frac{\partial^{2} \phi_{2}(t ; p)}{\partial t^{2}}-2 \phi_{1}(t ; p) \phi_{2}(t ; p)-\frac{\partial \phi_{2}(t ; p)}{\partial t} \phi_{3}(t ; p) \\
N_{3}\left[\phi_{1}(t ; p), \phi_{3}(t ; p)\right]=\frac{\partial \phi_{3}(t ; p)}{\partial t}+2 \phi_{1}(t ; p) \\
N_{4}\left[\phi_{1}(t ; p), \phi_{3}(t ; p), \phi_{4}(t ; p)\right]=\frac{\partial \phi_{4}(t ; p)}{\partial t}-2 \phi_{1}(t ; p) \phi_{3}(t ; p)+2 \frac{\partial \phi_{1}(t ; p)}{\partial t}
\end{gathered}
$$

Assuming $H(\tau)=1$, we use the above definitions to form the zero-order deformation equations

$$
\begin{array}{r}
(1-p) L\left[\varphi_{1}(t ; p)-f_{0}(t)\right]=p \hbar N_{1}\left[\varphi_{1}(t ; p), \varphi_{2}(t ; p), \varphi_{3}(t ; p)\right] \\
(1-p) L\left[\varphi_{2}(t ; p)-g_{0}(t)\right]=p \hbar N_{2}\left[\varphi_{1}(t ; p), \varphi_{2}(t ; p), \varphi_{3}(t ; p)\right] \\
(1-p) L\left[\varphi_{3}(t ; p)-h_{0}(t)\right]=p \hbar N_{3}\left[\varphi_{1}(t ; p), \varphi_{3}(t ; p)\right] \\
(1-p) L\left[\varphi_{4}(t ; p)-k_{0}(t)\right]=p \hbar N_{4}\left[\varphi_{1}(t ; p), \varphi_{3}(t ; p), \varphi_{4}(t ; p)\right]
\end{array}
$$

Obviously, when $p=0$ and $p=1$,

$$
\begin{array}{ll}
\phi_{1}(t ; 0)=f_{0}(t), & \phi_{1}(t ; 1)=f(t), \\
\phi_{2}(t ; 0)=g_{0}(t), & \phi_{2}(t ; 1)=g(t), \\
\phi_{3}(t ; 0)=h_{0}(t), & \phi_{3}(t ; 1)=h(t), \\
\phi_{4}(t ; 0)=k_{0}(t), & \phi_{4}(t ; 1)=k(t) .
\end{array}
$$

Differentiating the zero-order deformation Equations (37)-(40) $m$ times respecting $p$ and finally dividing by $m$ !, we have the $m$ th-order deformation equations as follows

$$
\begin{array}{r}
L\left[f_{m}(t)-x_{m} f_{m-1}(t)\right]=\hbar R_{m, 1}\left(\boldsymbol{f}_{m-1}, \boldsymbol{g}_{m-1}, \boldsymbol{h}_{m-1}\right) \\
L\left[g_{m}(t)-x_{m} g_{m-1}(t)\right]=\hbar R_{m, 2}\left(\boldsymbol{f}_{m-1}, \boldsymbol{g}_{m-1}, \boldsymbol{h}_{m-1}\right) \\
L\left[h_{m}(t)-x_{m} h_{m-1}(t)\right]=\hbar R_{m, 3}\left(\boldsymbol{f}_{m-1}, \boldsymbol{h}_{m-1}\right) \\
L\left[k_{m}(t)-x_{m} k_{m-1}(t)\right]=\hbar R_{m, 4}\left(\boldsymbol{f}_{m-1}, \boldsymbol{h}_{m-1}, \boldsymbol{k}_{m-1}\right)
\end{array}
$$

where

$$
\begin{gathered}
R_{m, 1}\left(\boldsymbol{f}_{m-1}, \boldsymbol{g}_{m-1}, \boldsymbol{h}_{m-1}\right)=\frac{\partial^{2} f_{m-1}(t)}{\partial t^{2}}+\sum_{n=0}^{m-1}\left[-f_{n}(t) f_{m-1-n}(t)+g_{n}(t) g_{m-1-n}(t)-\frac{\partial f_{n}(t)}{\partial t} h_{m-1-n}(t)\right], \\
R_{m, 2}\left(\boldsymbol{f}_{m-1}, \boldsymbol{g}_{m-1}, \boldsymbol{h}_{m-1}\right)=\frac{\partial^{2} g_{m-1}(t)}{\partial t^{2}}+\sum_{n=0}^{m-1}\left[-2 f_{n}(t) g_{m-1-n}(t)-\frac{\partial g_{n}(t)}{\partial t} h_{m-1-n}(t)\right],
\end{gathered}
$$




$$
\begin{gathered}
R_{m, 3}\left(\boldsymbol{f}_{m-1}, \boldsymbol{h}_{m-1}\right)=\frac{\partial h_{m-1}(t)}{\partial t}+2 f_{m-1}(t), \\
R_{m, 4}\left(\boldsymbol{f}_{m-1}, \boldsymbol{h}_{m-1}, \boldsymbol{k}_{m-1}\right)=\frac{\partial k_{m-1}(t)}{\partial t}+\sum_{n=0}^{m-1}\left[-2 f_{n}(t) h_{m-1-n}(t)\right]+2 \frac{\partial f_{m-1}(t)}{\partial t} .
\end{gathered}
$$

Using 10 terms in evaluating the approximate solution, and applying the padé approximation, we obtain

$$
\begin{aligned}
& f(t)_{[4,4]}=\left(0.51023 t-0.201277 t^{2}+0.0290848 t^{3}-0.00131806 t^{4}\right) / \\
&\left(1+0.585467 t+0.228364 t^{2}+0.047584 t^{3}+0.00768258 t^{4}\right), \\
& g(t)_{[4,4]}=\left(1+0.902069 t+0.00627076 t^{2}-0.103561 t^{3}+0.016795 t^{4}\right) / \\
&\left(1+1.51799 t+0.941231 t^{2}+0.306085 t^{3}+0.0566636 t^{4}\right), \\
& h(t)_{[4,4]}=\left(-0.51023 t^{2}+0.0169673 t^{3}-0.00727578 t^{4}\right) / \\
&\left(1+0.620046 t+0.218152 t^{2}+0.0425575 t^{3}+0.00483554 t^{4}\right), \\
& k(t)_{[4,4]}=\left(-1.02046 t-0.628178 t^{2}-0.222682 t^{3}-0.0285684 t^{4}\right) / \\
&\left(1+1.59553 t+1.37939 t^{2}+0.67214 t^{3}+0.181054 t^{4}\right) .
\end{aligned}
$$

Noting that, the different from all other analytical techniques such as the perturbation method, Adomian decomposition method, expansion method, and etc., the solutions given by the HAM contain auxiliary parameters $\hbar$ which could be used to control and adjust the convergence region and rate of the HAM series.

\section{Convergence of HAM}

It has been proven that, as long as a series solution given by the homotopy analysis method is converging, it is certainly one of the exact solutions. The solution series contains the auxiliary parameters $\hbar$ and which provides us with a simple way to adjust and control the convergence of the solution series. In general, by means of the so-called $\hbar$-curve, i.e., a curve of versus $\hbar$. As pointed by Liao [13], the valid region of $\hbar$ is a horizontal line segment. To see the range of admissible values of these parameters, the curves of $\hbar$ are plotted in Figure 1 for $f_{t t}(0), g_{t t t}(0), h_{t t}(0)$ and $k_{t t}(0)$ given by 10th-order approximation. For better presentation, these valid regions are listed in Table 1. A wide valid zone is evident in these figures ensuring convergence of the series. To choose optimal value of auxiliary parameter, the averaged residual errors [24] are defined as

$$
\begin{aligned}
& E_{f, m}=\frac{1}{\lambda} \sum_{i=0}^{\lambda}\left[N_{f}\left(\sum_{i=0}^{m} F_{i}(i \Delta x)\right)\right]^{2}, \\
& E_{g, m}=\frac{1}{\lambda} \sum_{i=0}^{\lambda}\left[N_{g}\left(\sum_{i=0}^{m} G_{i}(i \Delta x)\right)\right]^{2},
\end{aligned}
$$

Table 1 . The admissible values of $\hbar$ for each $\hbar$-curve .

\begin{tabular}{ccc}
\hline Number of Figure & Admissible Values of $\hbar$ & Optimal $\hbar$ \\
\hline 1(a) & $-1.4 \leq \hbar \leq-0.6$ & -0.999 \\
1(b) & $-1.4 \leq \hbar \leq-0.6$ & -0.910 \\
1(c) & $-1.5 \leq \hbar \leq-0.5$ & -1.002 \\
1(d) & $-1.2 \leq \hbar \leq-0.8$ & -1.060 \\
\hline
\end{tabular}




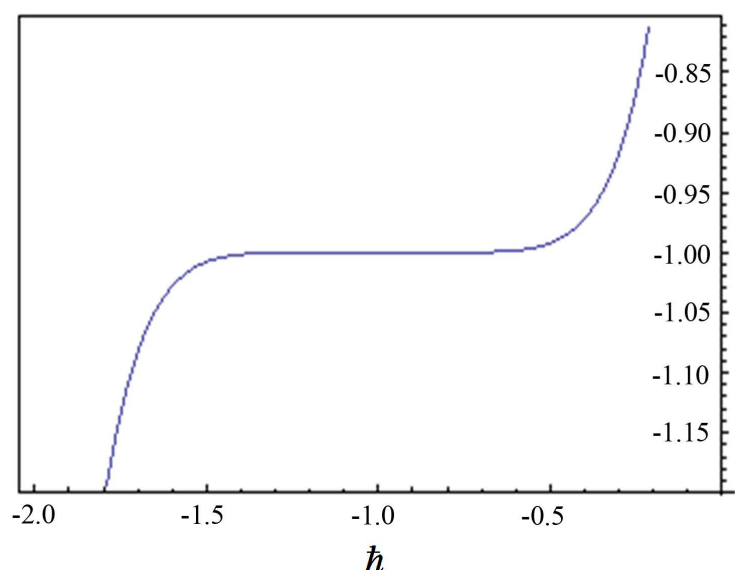

(a)

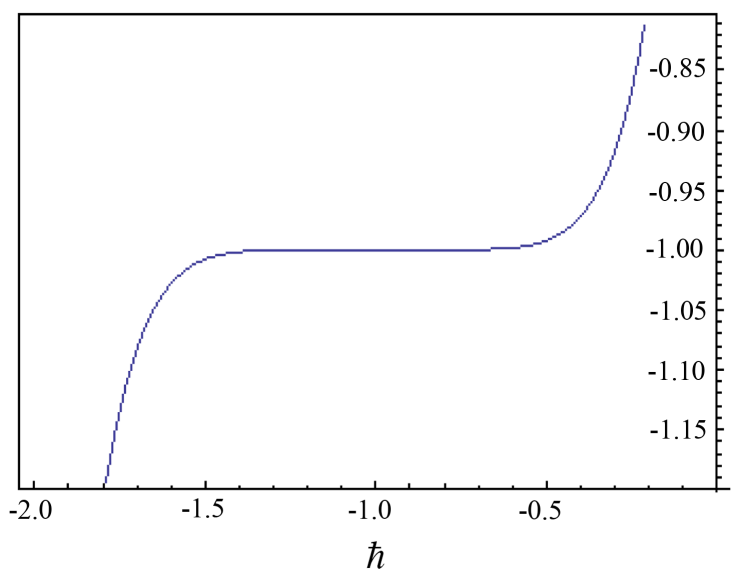

(c)

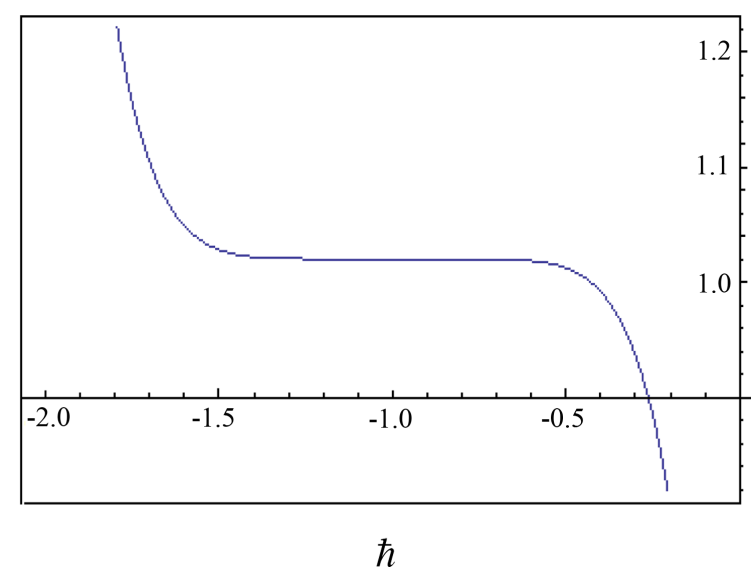

(b)

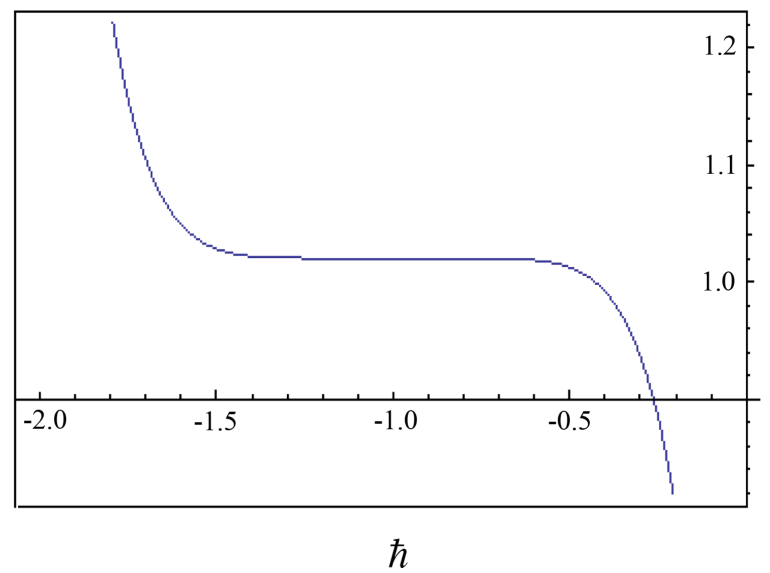

(d)

Figure 1. $\hbar$-curve of (a) $f_{t t}(0)$; (b) $g_{t t t}(0)$; (c) $h_{t t}(0)$ and (d) $k_{t t}(0)$ given by 10th order approximate solution.

$$
\begin{aligned}
& E_{h, m}=\frac{1}{\lambda} \sum_{i=0}^{\lambda}\left[N_{h}\left(\sum_{i=0}^{m} H_{i}(i \Delta x)\right)\right]^{2}, \\
& E_{k, m}=\frac{1}{\lambda} \sum_{i=0}^{\lambda}\left[N_{k}\left(\sum_{i=0}^{m} K_{i}(i \Delta x)\right)\right]^{2},
\end{aligned}
$$

where $\Delta x=10 / \lambda$ and $\lambda=20$. For a given order of approximation $m$, the optimal values of $\hbar$ is given by the minimum of $E_{m}$, corresponding to nonlinear algebraic equations

$$
\frac{\mathrm{d} E_{f, m}}{\mathrm{~d} \hbar}=0 \quad \text { or } \quad \frac{\mathrm{d} E_{g, m}}{\mathrm{~d} \hbar}=0 \quad \text { or } \quad \frac{\mathrm{d} E_{h, m}}{\mathrm{~d} \hbar}=0 \quad \text { or } \quad \frac{\mathrm{d} E_{k, m}}{\mathrm{~d} \hbar}=0 .
$$

It is noticed that the optimal value of $\hbar$ is replaced into the equations. Table 1 shows optimal values obtained for the auxiliary parameters $\hbar$. To see the accuracy of the solutions, the residual errors for the system are defined as

$$
\begin{gathered}
R E_{f}=F_{n}^{\prime \prime}(t)-F_{n}^{2}(t)+G_{n}^{2}(t)-F_{n}^{\prime}(t) H_{n}(t) \\
R E_{g}=G_{n}^{\prime \prime}(t)-2 F_{n}(t) G_{n}(t)-G_{n}^{\prime}(t) H_{n}(t) \\
R E_{H}=H_{n}^{\prime}(t)+2 F(t)
\end{gathered}
$$




$$
R E_{k}=K_{n}^{\prime}(t)-2 F_{n}(t) H_{n}(t)+2 F_{n}^{\prime}(t)
$$

where $F_{n}(t), G_{n}(t), H_{n}(t)$ and $K_{n}(t)$ are the HAM solutions for $f(\mathrm{t}), g(\mathrm{t}), h(\mathrm{t})$ and $k(\mathrm{t})$.

\section{Results and Discussion}

HAM-Padé, in this paper was used to find approximate solutions for nonlinear equations obtained by the steady, laminar, axially symmetric viscous flow near an infinite rotating disk. The accuracy of the method is appropriate and obtained results are close to the numerical solution. It is proper mentioning that the numerical results have been obtained using the fourth-order Runge-Kutta method along shooting method for satisfying boundary conditions at infinity. Figures 2-4 show the velocities in the $r, \theta$ and $z$ directions respectively. Figure 5 demonstrates distribution of pressure in the $z$ direction. In Table $1 \quad \hbar=-1$ is not a good approximation in every case. The results show that the HPM-Padé isonly valid for small values of independent variable.

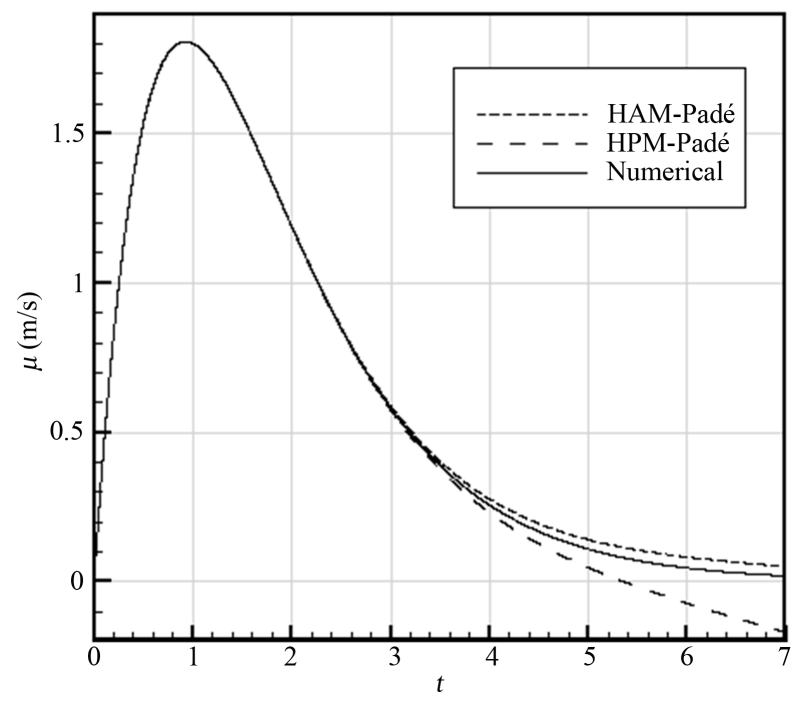

Figure 2. The velocity in $r$ direction for water with $r=0.01$, $\omega=1000 \mathrm{rad} / \mathrm{s}, \quad v=1.519 \times 10^{-6}\left(\mathrm{~m}^{2} / \mathrm{s}\right), \quad \rho=1000\left(\mathrm{~kg} / \mathrm{m}^{3}\right)$.

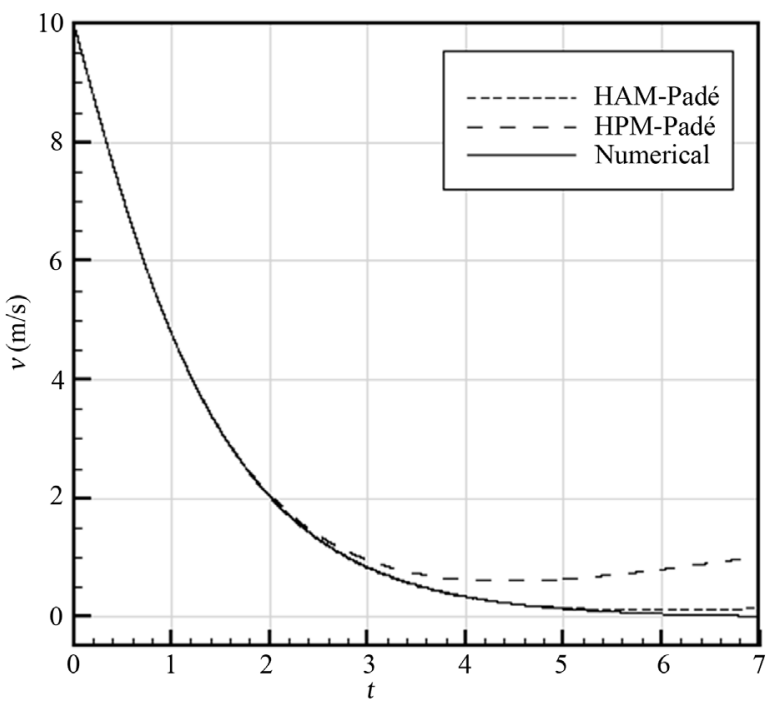

Figure 3. The velocity in $\theta$ direction for water with $r=0.01$, $\omega=1000 \mathrm{rad} / \mathrm{s}, \quad v=1.519 \times 10^{-6}\left(\mathrm{~m}^{2} / \mathrm{s}\right), \quad \rho=1000\left(\mathrm{~kg} / \mathrm{m}^{3}\right)$. 


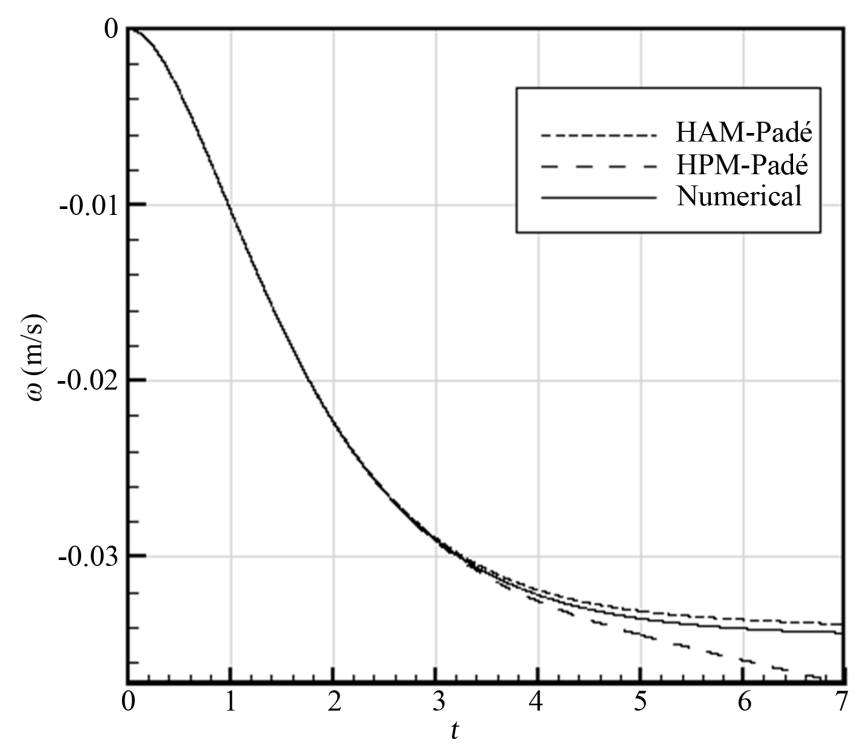

Figure 4. The velocity in $z$ direction for water with $r=0.01$, $\omega=1000 \mathrm{rad} / \mathrm{s}, \quad v=1.519 \times 10^{-6}\left(\mathrm{~m}^{2} / \mathrm{s}\right), \quad \rho=1000\left(\mathrm{~kg} / \mathrm{m}^{3}\right)$.

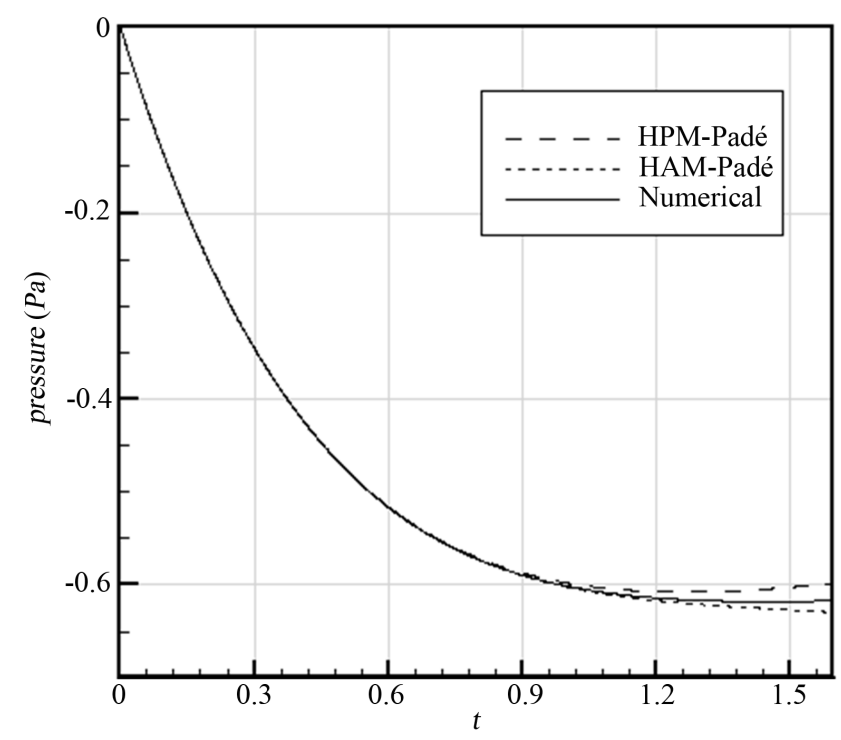

Figure 5. The pressure distribution in the $z$ direction for water with $r=0.01, \omega=1000 \mathrm{rad} / \mathrm{s}, v=1.519 \times 10^{-6}\left(\mathrm{~m}^{2} / \mathrm{s}\right)$, $\rho=1000\left(\mathrm{~kg} / \mathrm{m}^{3}\right)$.

\section{Conclusions}

In this paper, homotopy analysis method (HAM) and Padé approximant were considered for finding analytical solution of three-dimensional viscous flow near an infinite rotating disk which is a well-known classical problem in fluid mechanics. A comparison of HAM-Padé and HPM-Padé is made. Unlike perturbation method, the HAM does not depend on any small physical parameters. Thus, it is valid for both weak and strong nonlinear problems. Besides, the HAM provides us with a convenient way of controlling the convergence of approximation series, by means of auxiliary parameter, which is a fundamental qualitative difference in analysis between the HAM and other methods. Also the figures show that the HAM-Padé is a powerful mathematical tool for solving the system of non-linear partial differential equations having wide applications in engineering. 
Finally, the recent appearance of nonlinear differential equations as models in some fields of applied mathematics makes it necessary to investigate innovative methods of solution for such equations.

\section{References}

[1] Von Karman, T. (1921) Uberlaminare und turbulence reibung. ZAMM, 1, 52-233.

[2] Rashidi, M.M. and Shahmohamadi, H. (2009) Analytical Solution of Three-Dimensional Navier-Stokes Equations for the Flow near an Infinite Rotating Disk. Communications in Nonlinear Science and Numerical Simulation, 14, 29993006. http://dx.doi.org/10.1016/j.cnsns.2008.10.030

[3] Ablowitz, M.J. and Clarkson, P.A. (1990) Solitons, Nonlinear Evolution Equations and Inverse Scattering Transform. Cambridge University Press, Cambridge.

[4] Fan, E. (2000) Extentedtanh-Function Method and Its Applications to Nonlinear Equations. Physics Letters A, 277, 212-218. http://dx.doi.org/10.1016/S0375-9601(00)00725-8

[5] Wazwaz, A.M. (2006) The Tanh and the Sine-Cosine Methods for a Reliable Treatment of the Modified Equal Width Equation and Its Variants. Communications in Nonlinear Science and Numerical Simulation, 11, 148-160. http://dx.doi.org/10.1016/j.cnsns.2004.07.001

[6] Fan, E. and Zhang, H. (1998) A Note on the Homogeneous Balance Method. Physical Letters A, 246, 403-406. http://dx.doi.org/10.1016/S0375-9601(98)00547-7

[7] He, J.H. and Wu, X.H. (2006) Exp-Function Method for Nonlinear Wave Equations. Chaos Solitons Fractals, 30, 700708. http://dx.doi.org/10.1016/j.chaos.2006.03.020

[8] Rashidi, M.M., Shahmohamadi, H. and Domairry, G. (2011) Variational Iteration Method for Solving Three-Dimensional Navier-Stokes Equations of Flow between Two Stretchable Disks. Numerical Methods for Partial Differential Equations, 27, 292-301. http://onlinelibrary.wiley.com/doi/10.1002/num.20522/pdf http://dx.doi.org/10.1002/num.20522

[9] Rashidi, M.M., Ganji, D.D. and Shahmohamadi, H. (2011) Variational Iteration Method for Two-Dimensional Steady Slip Flow in Micro-Channels. Archive of Applied Mechanics, 81, 1597-1605. http://link.springer.com/article/10.1007\%2Fs00419-010-0504-X http://dx.doi.org/10.1007/s00419-010-0504-x

[10] Shahmohamadi, H., Rashidi, M.M. and Anwar Bég, O. (2012) A New Technique for Solving Steady Flow and Heat Transfer from a Rotating Disk in High Permeability Media. International Journal of Applied Mathematics and Mechanics, 8, 1-17. http://ijamm.bc.cityu.edu.hk/ijamm/outbox/Y2012V8N7P1C37192935.pdf

[11] Shahmohamadi, H. and Rashidi, M.M. (2010) A Novel Solution for the Glauert-Jet Problem by Variational Iteration Method-Padé Approximant. Mathematical Problems in Engineering, 2010, Article ID: 501476.

http://dx.doi.org/10.1155/2010/501476

[12] Shahmohamadi, H. and Rashidi, M.M. (2011) Explicit Solutions for Steady Three-Dimensional Problem of Condensation Film on Inclined Rotating Disk. International Journal of Fluid Mechanics Research, 38, 353-365. http://www.dl.begellhouse.com/journals/71cb29ca5b40f8f8,3b02a11311f01f8d,714ff56d76778e5a.html http://dx.doi.org/10.1615/InterJFluidMechRes.v38.i4.50

[13] Liao, S.J. (1992) The Proposed Homotopy Analysis Technique for the Solution of Nonlinear Problems. PhD Thesis, Shanghai Jiao Tong University, Shanghai.

[14] Liao, S.J. (2003) Beyond Perturbation: Introduction to Homotopy Analysis Method. Chapman \& Hall/CRC, Boca Raton. http://dx.doi.org/10.1201/9780203491164

[15] He, J.H. (2000) A Coupling Method for Homotopy Technique and Perturbation Technique for Nonlinear Problem. International Journal of Non-Linear Mechanics, 35, 37-43. http://dx.doi.org/10.1016/S0020-7462(98)00085-7

[16] Liao, S.J. (2005) Comparison between the Homotopy Analysis Method and Homotopy Perturbation Method. Applied Mathematics and Computation, 169, 1186-1194. http://dx.doi.org/10.1016/j.amc.2004.10.058

[17] Sajid, M., Hayat, T. and Asghar, S. (2007) Comparison between the HAM and HPM Solutions of Tin Film Flows of Non-Newtonian Fluids on a Moving Belt. Nonlinear Dynamics, 50, 27-35. http://dx.doi.org/10.1007/s11071-006-9140-y

[18] Abbasbandy, S. (2007) The Application of Homotopy Analysis Method to Solve a Generalized Hirota-Satsuma Coupled KdV Equation. Physics Letters A, 361, 478-483. http://dx.doi.org/10.1016/j.physleta.2006.09.105

[19] Sajid, M. and Hayat, T. (2008) Comparison of HAM and HPM Methods in Nonlinear Heat Conduction and Convection Equations. Nonlinear Analysis: Real World Applications, 9, 2296-2301.

http://dx.doi.org/10.1016/j.nonrwa.2007.08.007 
[20] Shahmohamadi, H. (2012) Analytic Study on Non-Newtonian Natural Convection Boundary Layer Flow with Variable Wall Temperature on a Horizontal Plate. Meccanica, 47, 1313-1323.

http://link.springer.com/article/10.1007\%2Fs11012-011-9515-0 http://dx.doi.org/10.1007/s11012-011-9515-0

[21] Shahmohamadi, H. (2011) Reliable Treatment of a New Analytical Method for Solving MHD Boundary-Layer Equations. Meccanica, 46, 921-933. http://link.springer.com/article/10.1007\%2Fs11012-010-9350-8 http://dx.doi.org/10.1007/s11012-010-9350-8

[22] Rashidi, M.M., Shahmohamadi, H. and Dinarvand, S. (2008) Analytic Approximate Solutions for Unsteady Two-Dimensional and Axisymmetric Squeezing Flows between Parallel Plates. Mathematical Problems in Engineering, 2008, Article ID: 935095. http://dx.doi.org/10.1155/2008/935095

[23] Ismail, H.N.A. and Abde Rabboh, A.A. (2004) A Restrictive Padé Approximation for the Solution of the Generalized Fisher and Burger-Fisher Equations. Applied Mathematics and Computation, 154, 203-213. http://dx.doi.org/10.1016/S0096-3003(03)00703-3

[24] Liao, S.J. (2010) An Optimal Homotopy-Analysis Approach for Strongly Nonlinear Differential Equations. Communications in Nonlinear Science and Numerical Simulation, 15, 2003-2016. http://dx.doi.org/10.1016/j.cnsns.2009.09.002 